\title{
Pyrrolizilactone, a new pyrrolizidinone metabolite produced by a fungus
}

\author{
Toshihiko Nogawa ${ }^{1}$, Makoto Kawatani ${ }^{2}$, Masakazu Uramoto ${ }^{2}$, Akiko Okano ${ }^{1}$, Harumi Aono², \\ Yushi Futamura $^{2}$, Hiroyuki Koshino ${ }^{3}$, Shunji Takahashi ${ }^{4}$ and Hiroyuki Osada ${ }^{1,2}$
}

The Journal of Antibiotics (2013) 66, 621-623; doi:10.1038/ja.2013.55; published online 29 May 2013

Keywords: cytotoxicity; fungal metabolite; isolation; pyrrolizidinone; structure elucidation

In the course of our screening program to find structurally unique metabolites from microorganisms on the basis of spectral data collected through LC/MS analysis, a new pyrrolizidinone metabolite, pyrrolizilactone (1) (Figure 1), was discovered and isolated from an uncharacterized fungus. The structure of 1 was determined from spectroscopic results. Compound $\mathbf{1}$ showed moderate cytotoxic activity against HL-60 and HeLa cells.

Microorganisms have a tremendous capacity for producing structurally diverse metabolites, which show various activities. ${ }^{1}$ They are important sources of pharmaceutical leads and therapeutic agents, ${ }^{2,3}$ and are also used as bioprobes in chemical biology for the exploration of biological functions. ${ }^{4,5}$ To search for and discover such structurally unique metabolites efficiently and rapidly, we have constructed a microbial metabolite fraction library with a spectral database on the basis of photodiode array detectorattached LC/MS analysis. 6,7 Through our methodology for the construction of this fraction library, we discovered and identified a 16 -membered macrolactam with an unusual $\beta$-keto-amide moiety, verticilactam, ${ }^{8} 6,6$-spiroacetal polyketide, spirotoamides, A and B, ${ }^{9}$ the new fraquinocins, I and $\mathrm{J},{ }^{10}$ and 6 -dimethylallylindole-3carbaldehyde. ${ }^{11}$ These results demonstrate the advantage of the fraction library in isolating novel metabolites from natural sources. We report herein the isolation of a novel compound from a fungi fraction library.
An 18-liter culture broth of an uncharacterized fungus was cultivated to obtain $16.1 \mathrm{~g}$ of an ethyl acetate-soluble extract. This was separated into eight fractions through a silica-gel column chromatography, with a stepwise gradient of $\mathrm{CHCl}_{3} / \mathrm{MeOH}$. The second fraction eluted with $\mathrm{CHCl}_{3} / \mathrm{MeOH}$ (100:1) was further separated by chromatography using a Sephadex LH-20 column with $\mathrm{CHCl}_{3} / \mathrm{MeOH}$ (1:1) to afford three fractions. The second fraction, showing an unidentified peak in LC/MS analysis, was purified by $\mathrm{C}_{18^{-}}$ HPLC to afford a colorless amorphous solid (1, $5.5 \mathrm{mg})$. Colorless amorphous; $[\alpha]_{589^{26}}+5.9^{\circ}(c 0.08, \mathrm{MeOH}) ; \mathrm{UV}(\mathrm{MeOH}) \lambda_{\max }(\log \varepsilon)$ 209 (3.34), $236(3.01) \mathrm{nm}$; IR (ATR) $v_{\max }\left(\mathrm{cm}^{-1}\right) 3410,2920,2875$, $1790,1715,1685,1575,1450,1375,1335,1280,1160,1110,1020 ;{ }^{1} \mathrm{H}$ NMR and ${ }^{13} \mathrm{C}$ NMR data, see Table 1; HRESIMS $m / z$ : 416.2433 $[\mathrm{M}+\mathrm{H}]^{+}$(calcd for $\mathrm{C}_{24} \mathrm{H}_{34} \mathrm{NO}_{5}$ : 416.2437).

Compound 1 had the molecular formula $\mathrm{C}_{24} \mathrm{H}_{33} \mathrm{NO}_{5}$, as determined by HRESIMS. The IR spectrum implied the presence of hydroxyl $\left(3410 \mathrm{~cm}^{-1}\right)$ and carbonyl $\left(1685,1715\right.$ and $\left.1790 \mathrm{~cm}^{-1}\right)$ groups. The ${ }^{1} \mathrm{H}$ NMR spectrum showed five methyl signals, which included a singlet, three doublets and a doublet of doublet (1.71 p.p.m., dd, $J=0.9,0.9 \mathrm{~Hz}$ ) branched at an $\mathrm{sp}^{2}$ carbon, and an olefin signal (5.12 p.p.m., br s), suggesting that 1 contained a double bond (Supplementary Figure S1). The ${ }^{13} \mathrm{C}$ NMR spectrum showed 24 signals including five methyls, four methylenes, nine methines (including oxygenated and olefin carbons: 81.6 and 131.3 p.p.m.),

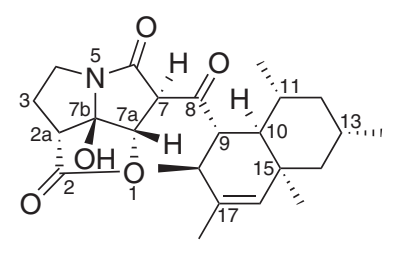

1

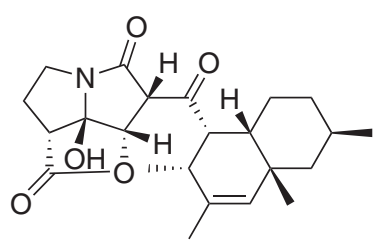

CJ-16264

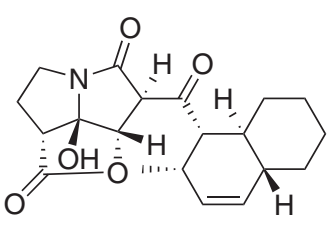

UCS1025A

Figure 1 Structures of compound 1 and related compounds.

${ }^{1}$ Chemical Biology Research Group, RIKEN CSRS, Saitama, Japan; ${ }^{2}$ Antibiotics Laboratory, RIKEN, Saitama, Japan; ${ }^{3}$ RIKEN Global Research Cluster, RIKEN, Saitama, Japan and ${ }^{4}$ Natural Product Biosynthesis Research Unit, RIKEN CSRS, Saitama, Japan

Correspondence: Dr H Osada, Antibiotics Laboratory, RIKEN, 2-1 Hirosawa, Wako, Saitama 351-0198, Japan.

E-mail: hisyo@riken.jp

Received 9 April 2013; revised 1 May 2013; accepted 7 May 2013; published online 29 May 2013 
Table $1{ }^{1} \mathrm{H}$ and ${ }^{13} \mathrm{C}$ NMR chemical shifts with $\mathrm{HMBC}$ and NOESY correlations in $\mathrm{CDCl}_{3}$

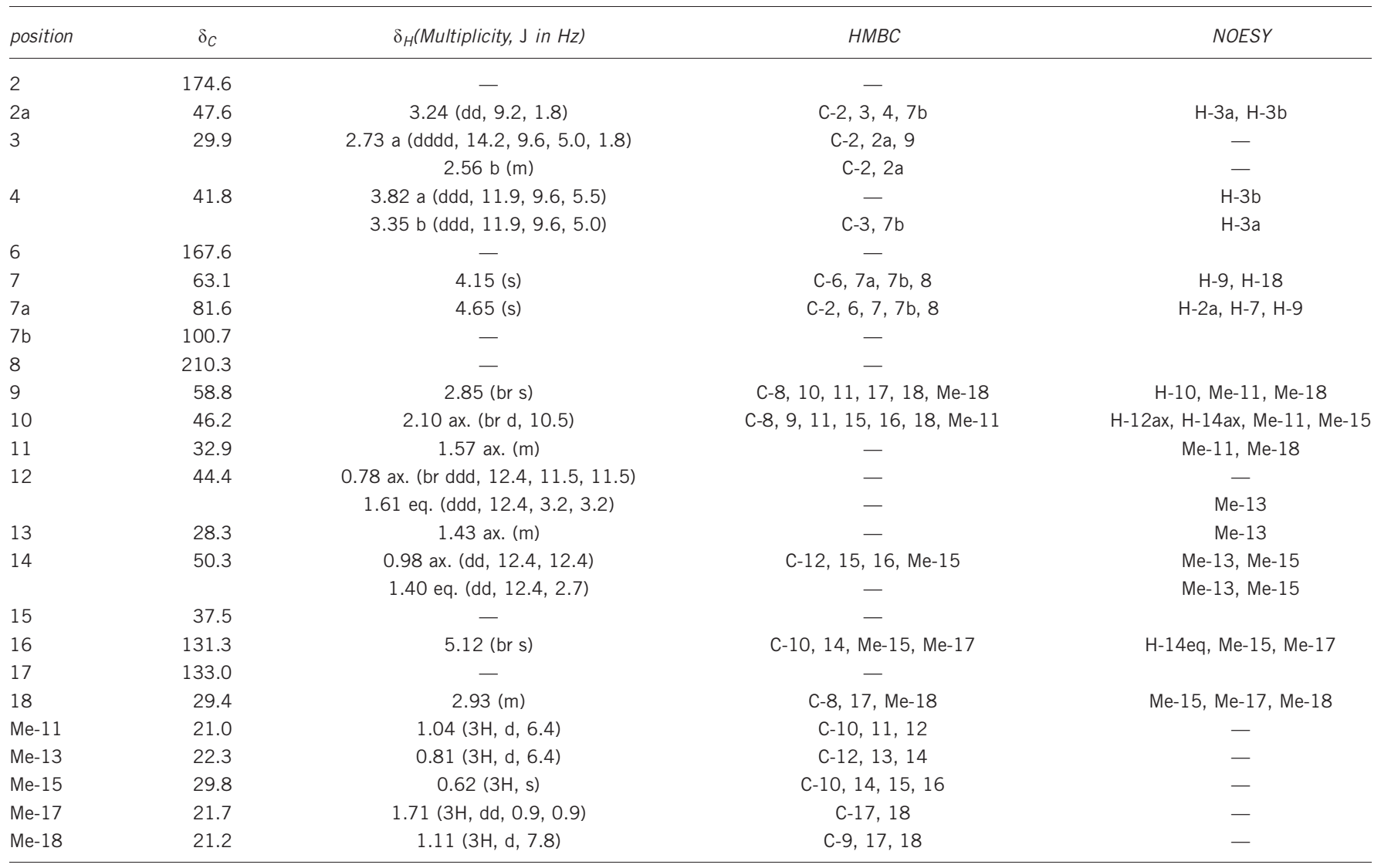

${ }^{1} \mathrm{H}$ and ${ }^{13} \mathrm{C}$ NMR spectra were recorded at 500 and $125 \mathrm{MHz}$, respectively.

and six quaternary carbons including an olefin carbon (133.0 p.p.m.) and three carbonyl ones (167.6, 174.6 and 210.3 p.p.m.), one of which was a ketone (Supplementary Figure S2). These were confirmed by the ${ }^{13} \mathrm{C}$ DEPT experiment and HSQC spectrum (Supplementary Figures S3 and S4). The spectrum also included an unusual quaternary carbon at 100.7 p.p.m., implied to bear both oxygen and nitrogen atoms or two oxygen atoms. These data and the index of hydrogen deficiency of nine suggested that compound $\mathbf{1}$ consisted of a five-ring system with three carbonyls and one double bond. The planar structure was established through the interpretation of the 2D NMR spectra (Figure 2a). The connections between protons and carbons were confirmed by correlations in the HSQC spectrum. Two proton-spin systems from $\mathrm{H}-2 \mathrm{a}$ to $\mathrm{H}-4$ and from Me-18 to H-14 with methyl branches at C-11 and C-13 were constructed from the correlations in the DQF-COSY and HSQCTOCSY spectra (Supplementary Figures S5 and S6). The overall structure was established from the long-range correlations in the HMBC spectrum (Supplementary Figure S7). The HMBC correlations from Me-15 to C-10, C-14, C-15 and C-16 showed the attachment of Me-15 at C-15, which was connected to C-10 to construct a cyclohexane substructure, and connectivities from C-14 to C-16. These were confirmed by the HMBC correlations from $\mathrm{H}-10$ to $\mathrm{C}-15$ and $\mathrm{C}-16$, and from $\mathrm{H}-14$ to $\mathrm{C}-15$ and C-16. Me-17, which should be branched at an $\mathrm{sp}^{2}$ carbon, showed HMBC correlations to C-16, C-17 and $\mathrm{C}-18$, suggesting a methyl branch at $\mathrm{C}-17$ and connectivities from $\mathrm{C}-16$ to C-18. These results in combination with the DQF-COSY result indicated a pentamethyl-octahydronaphthalene structure; this was confirmed by HMBC correlations from H-9 to C-15 and C-17, and from both $\mathrm{H}-18$ and Me-18 to C-17.
A pyrrolizidinone moiety fused with $\gamma$-lactone system was confirmed by HMBC correlations and NMR chemical shift values with the consideration of the index of hydrogen deficiency, which suggested the remaining substructure required a three-ring system. The HMBC correlations from $\mathrm{H}-2 \mathrm{a}$ and $\mathrm{H}-3$ to a carbonyl carbon C-2 and from $\mathrm{H}-2 \mathrm{a}$ to $\mathrm{C}-7 \mathrm{~b}$ having the unusual ${ }^{13} \mathrm{C}$ NMR chemical shift value of 100.7 p.p.m. were observed. The C-7b was also correlated from $\mathrm{H}-7$ and $\mathrm{H}-7 \mathrm{a}$ in the HMBC spectrum, both of which showed HMBC correlations to another carbonyl carbon C-6. These HMBC correlations and the connectivities from $\mathrm{H}-2 \mathrm{a}$ to $\mathrm{H}-4$ was confirmed by DQF-COSY, as described above, were allowing the connectivities between C-4 and C-6 with a branch of the carbonyl carbon C-2 at C-2a position. The connection between C-7 and C-7a, both of which were observed as a singlet signal in the ${ }^{1} \mathrm{H}$ NMR spectrum and did not show COSY correlation, was confirmed by an HMBC correlation from $\mathrm{H}-7$ to $\mathrm{C}-7 \mathrm{a}$ and NOE between $\mathrm{H}-7$ and $\mathrm{H}-7 \mathrm{a}$ in the phasesensitive NOESY spectrum (Supplementary Figure S8). An HMBC correlation from $\mathrm{H}-7 \mathrm{a}$ to $\mathrm{C}-2$ implied the presence of a ring system, and it was confirmed as a $\gamma$-lactone by the ${ }^{13} \mathrm{C}$ NMR chemical shift values of C-2 and C-7a (174.6 and 81.6 p.p.m., respectively). Based on the above results and the consideration of molecular formula, the $\mathrm{C}-7 \mathrm{~b}$ was supposed to have both oxygen and nitrogen atoms. The ${ }^{13} \mathrm{C}$ NMR chemical shift values of C-4 and C-6 (41.8 and 167.6 p.p.m., respectively) and the substructure being a tricyclo-system allowed the construction of a pyrrolizidinone skeleton, which were also confirmed by an $\mathrm{HMBC}$ correlation from $\mathrm{H}-4$ to $\mathrm{C}-7 \mathrm{~b}$. The pyrrolizidinone substructure was connected to the decalin moiety through the ketone C-8, which was confirmed by HMBC correlations from H-7, H-7a, 
a

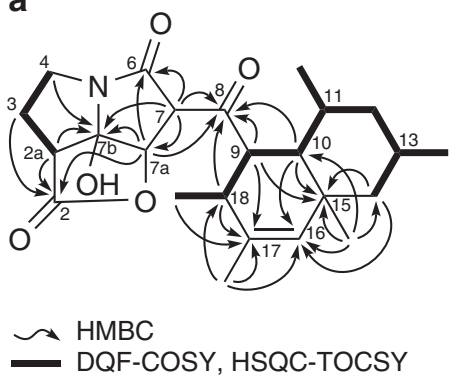

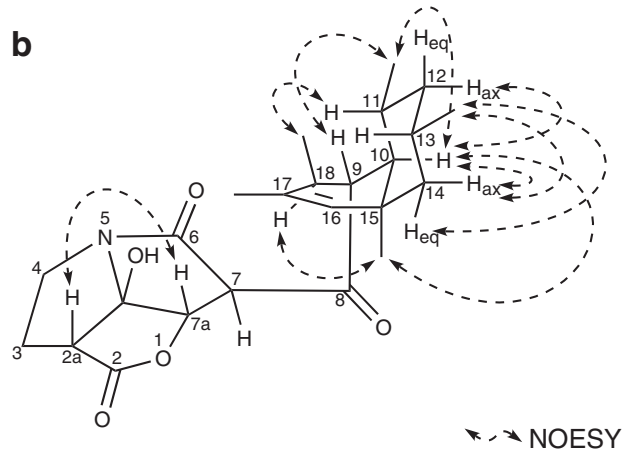

Figure 2 Selected DQF-COSY and HMBC correlations (a) and selected NOESY correlations (b).

H-9, H-10 and H-18 to the remaining carbonyl carbon at 210.3 p.p.m. Therefore, the planar structure of compound $\mathbf{1}$ was determined.

The relative stereochemistry was examined by the phase-sensitive NOESY spectrum (Figure 2b). H-10 showed NOEs with $\mathrm{H}-12$ at 0.78 p.p.m. and $\mathrm{H}-14$ at 0.98 p.p.m., and these correlations suggested that the cyclohexane had a chair conformation and all three protons were assigned as axials. H-10 also had NOESY correlations with Me-11 and Me-15, allowing the assignments of their equatorial-orientations on the cyclohexane part, and a cis-configuration of the decalin moiety, which was also confirmed by NOEs between H-11 and Me-18, between Me-11 and H-9, and between Me-15 and H-18. These NOEs were also confirmed by $\beta$-orientation of Me- 18 and $\alpha$-orientation of the pyrrolizidinone moiety at C-9 position. Me-13 was assigned as an equatorial by the large coupling constants of ${ }^{3} J_{\mathrm{H}-12 \mathrm{ax} / \mathrm{H}-13}$ and ${ }^{3} J_{\mathrm{H}-13 / \mathrm{H}-14 \mathrm{ax}}$, which suggested $\mathrm{H}-13$ was an axial. It was also confirmed by the NOEs with both $\mathrm{H}$-14axial and $\mathrm{H}$-14equatorial. In the pyrrolizidinone moiety, an NOE between $\mathrm{H}-2 \mathrm{a}$ and $\mathrm{H}-7 \mathrm{a}$ was observed suggesting $\beta$-orientations of them, which also allowed the assignment of $\beta$-orientation of the hydroxyl group at C-7b. H-7 was assigned as $\alpha$-orientation by the dihedral angle of $\mathrm{H}-7-\mathrm{C}-7-\mathrm{C}-7 \mathrm{a}-\mathrm{H}-$ 7 a was approximately $90^{\circ}$, which were implied from the weak vicinal coupling between $\mathrm{H}-7$ and H-7a. Based on all the above results, the structure of compound $\mathbf{1}$ was determined as shown in Figure 1 and designated as pyrrolizilactone.

The configuration between the decalin and pyrrolizidinone moieties could not be determined by NOESY spectrum due to free rotation at C-7 and C-9 positions, but NOEs between $\mathrm{H}-7$ and H-9 and $\mathrm{H}-7$ and $\mathrm{H}-18$ were observed (Table 1). Therefore, there are four possible stereo-structures, which include two enantiomers and two diastereomers by the same relative stereochemistry.

Cytotoxic and antibacterial activities of compound $\mathbf{1}$ were evaluated in vitro. Compound 1 showed moderate cytotoxicities against human promyelocytic leukemia cell line HL-60 and human cervical cancer cell line $\mathrm{HeLa}$ with $\mathrm{IC}_{50}$ values of 1.1 and $3.1 \mu \mathrm{g} \mathrm{ml}^{-1}$, respectively. In contrast, it did not show antibacterial activity against Escherichia coli up to $30 \mu \mathrm{g} \mathrm{ml}^{-1}$. Two compounds, CJ-16264 ${ }^{12}$ and UCS1025A, ${ }^{13}$ have been reported in the same class of metabolites (Figure 1), and show broad antibacterial activities against Grampositive bacteria. It is worth evaluating such activities in future work. We have also observed morphological changes on cancer cell lines, and will report the findings elsewhere.

Compound $\mathbf{1}$ had a tricyclic skeleton composed of a pyrrolizidinone moiety fused with a $\gamma$-lactone, which was connected to a decalin moiety by a ketone. As mentioned above, only two compounds, UCS1025A and CJ-16264, have been reported in the same class of fungal metabolites, and the differences between them in terms of their planar structure lie in the substitution pattern of methyl groups on the decalin moiety. That of $\mathbf{1}$ was highly substituted with five methyl groups. UCS1025A, on the other hand, had only one methyl group. In terms of the stereochemistry, the pyrrolizidinone moiety had the same relative configuration as UCS1025A, with a trans configuration at H-7 and H-7a. The structure of CJ-16264 was shown to have a cisconfiguration at $\mathrm{H}-7$ and $\mathrm{H}-7 \mathrm{a} .{ }^{12}$ This could be a mistake, because Sugie et al. ${ }^{12}$ determined the dihedral angle of $\mathrm{H}-7-\mathrm{C}-7-\mathrm{C}-7 \mathrm{a}-\mathrm{H}-7 \mathrm{a}$ to be $84.9^{\circ}$ through molecular mechanics calculations, which clearly suggests a trans configuration. The decalin unit of 1 had a cis-configuration, which is consistent with CJ-16264 but not with UCS1025A, which has a trans configuration.

\section{ACKNOWLEDGEMENTS}

We thank Drs T Nakamura and Y Hongo (RIKEN) for the HRESIMS measurements. We also thank Dr T Shimizu (RIKEN) for useful discussions on the structure. This work was supported in part by Technology of Japan, the Program for Promotion of Basic and Applied Researches for Innovations in Bio-oriented Industry, and a Health and Labor Sciences Research Grant.

1 Osada, H. An overview on the diversity of actinomycete metabolites. Actinomycetol 15, 11-14 (2001)

2 Newman, D. J. \& Cragg, G. M. Natural products as sources of new drugs over the 30 years from 1981 to 2010. J. Nat. Prod. 75, 311-335 (2012).

3 Dobson, C. M. Chemical space and biology. Nature 432, 824-828 (2004).

4 Osada, H. Bioprobes (ed. Osada, H.) 1-14 (Springer, Berlin, 2000).

5 Osada, H. Chemical Biology Techniques and Applications (ed. Osada, H.) 1-10 (Wiley, New Jersey, 2009).

6 Osada, H. \& Nogawa, T. Systematic isolation of microbial metabolites for natural products depository (NPDepo). Pure Appl. Chem. 84, 1407-1420 (2012).

7 Kato, N., Takahashi, S., Nogawa, T., Saito, T. \& Osada, H. Construction of a microbial natural product library for chemical biology studies. Curr. Opin. Chem. Biol. 16, 101-108 (2012).

8 Nogawa, T. et al. Verticilactam, a new macrolactam isolated from a microbial metabolite fraction library. Org. Lett. 12, 4564-4567 (2010).

9 Nogawa, T. et al. Spirotoamides A and B, novel 6,6-spiroacetal polyketides isolated from a microbial metabolite fraction library. J. Antibiot. 65, 123-128 (2012).

10 Panthee, S. et al. Furaquinocins I and J: novel polyketide isoprenoid hybrid compounds from Streptomyces reveromyceticus SN-593. J. Antibiot. 64, 509-513 (2011).

11 Takahashi, S. et al. Biochemical characterization of a novel indole prenyltransferase from Streptomyces sp. SN-593. J. Bacteriol 192, 2839-2851 (2010).

12 Sugie, Y. et al. New pyrrolizidinone antibiotics CJ-16,264 and CJ-16,367. J. Antibiot. 54, 917-925 (2001).

13 Agatsuma, T. et al. UCS1025A and B, new antitumor antibiotics from the fungus Acremonium species. Org. Lett 4, 4387-4390 (2002). 